\title{
Precision Tests of the Standard Model
}

\author{
Martin W. Grünewald* \\ University College Dublin, Ireland \\ E-mail: Martin.Grunewald@cern.ch
}

\begin{abstract}
Recent published and preliminary precision electroweak measurements are reviewed, including new results on the mass of the top quark and mass and width of the $\mathrm{W}$ boson. The experimental results are compared with the predictions of the Standard Model and are used to constrain its free parameters, notably the mass of the Higgs boson. The agreement between measurements and expectations from theory is discussed.
\end{abstract}

International Europhysics Conference on High Energy Physics

July 21st - 27th 2005

Lisboa, Portugal

${ }^{*}$ Speaker. 


\section{Introduction}

On the level of realistic observables such as measured cross sections, ratios and asymmetries, the electroweak precision data consist of over thousand measurements with partially correlated statistical and systematic uncertainties. This large set of results is reduced to a more manageable set of 17 precision results, so-called pseudo observables, in a largely model-independent procedure, by the LEP and Tevatron Electroweak Working Groups. The pseudo observables updated for this conference are briefly reviewed and Standard Model analyses [1] are performed, where the hadronic vacuum polarisation at the $\mathrm{Z}$ pole and "constants" such as the Fermi constant $G_{\mathrm{F}}$ are used as well.

\section{Measurements}

About 3/4 of all pseudo observables arise from measurements performed in electron-positron collisions at the $\mathrm{Z}$ resonance, by the SLD experiment and the LEP experiments ALEPH, DELPHI, L3 and OPAL. The Z-pole observables are: 5 observables describing the Z lineshape and leptonic forward-backward asymmetries, 2 observables describing polarised leptonic asymmetries measured by SLD with polarised beams and at LEP exploiting tau polarisation, 6 observables describing $\mathrm{b}$ - and c-quark production at the $\mathrm{Z}$ pole, and finally the inclusive hadronic charge asymmetry. The Z-pole results and their combinations are final and by now published [2]. The remaining pseudo observables are: the mass and total width of the $\mathrm{W}$ boson measured by CDF and $\mathrm{D} \emptyset$ at the Tevatron and by the four LEP-II experiments, and the top quark mass measured at the Tevatron.

\section{Mass of the Top Quark}

Ten years ago the Tevatron experiments CDF and $\mathrm{D} \emptyset$ discovered the top quark in protonantiproton collisions at $1.8 \mathrm{TeV}$ centre-of-mass energy, by observing the reaction $\mathrm{p} \overline{\mathrm{p}} \rightarrow \mathrm{t} \overline{\mathrm{t}} X, \mathrm{t} \overline{\mathrm{t}} \rightarrow$ $\mathrm{b} \overline{\mathrm{b}} \mathrm{W}^{+} \mathrm{W}^{-}$. The published results based on data collected during Run-I (1992-1996) and the preliminary results based on Run-II data (since 2001) presented at this conference [3] are combined by the Tevatron Electroweak Working [4]: $M_{\mathrm{t}}=172.7 \pm 1.7$ (stat.) \pm 2.4 (syst.) GeV.

\section{Mass and Width of the $W$ boson}

Final results on $M_{\mathrm{W}}$ and $\Gamma_{\mathrm{W}}$ from CDF and D $\emptyset$ are available for the complete Run-I data set and are combined taking correlations properly into account [5]. No results are available for Run-II data yet. The results from ALEPH, DELPHI and L3 are preliminary, while OPAL has recently published final results for their complete LEP-II data set [6]. The combined results of the Tevatron $\left(M_{\mathrm{W}}=80.452 \pm 0.059 \mathrm{GeV}\right)$ and LEP-II $\left(M_{\mathrm{W}}=80.392 \pm 0.039 \mathrm{GeV}\right)$ are in very good agreement.

\section{Z Decays to b and c Quarks}

The heavy-flavour results at the Z-pole were the last precision electroweak Z-pole results to become final. Details on the various heavy-flavour measurements at the $\mathrm{Z}$ pole are given in [2]. The combination has a rather low $\chi^{2}$ of 53 for $(105-14)$ degrees of freedom: all forward-backward asymmetries are very consistent, and their combination is still statistics limited. The combined values for $A_{\mathrm{fb}}^{0, \mathrm{~b}}$ and $\mathrm{A}_{\mathrm{fb}}^{0, \mathrm{c}}$ are compared to the $\mathrm{SM}$ expectation in Figure 1 (left), showing that $\mathrm{A}_{\mathrm{fb}}^{0, \mathrm{~b}}$ agrees well with the SM expectation for an intermediate Higgs-boson mass of a few hundred $\mathrm{GeV}$. The mutual consistency of the measurements of $A_{b}, A_{f b}^{0, b}=(3 / 4) A_{e} A_{b}$ and $A_{\ell}$ assuming lepton universality is shown in Figure 1 (right). Compared to the experimental uncertainties, the SM predictions are nearly constant in $\mathrm{A}_{q}$, in contrast to the situation for $\mathrm{A}_{\ell}$. This is a consequence of the SM values of electric charge and iso-spin of quarks. 


\section{Effective Electroweak Mixing Angle}

Assuming the SM structure of the effective coupling constants, the measurements of the various asymmetries are compared in terms of $\sin ^{2} \theta_{\text {eff }}^{\text {lept }}$ in Figure 2 (left). The average of all six $\sin ^{2} \theta_{\text {eff }}^{\text {lept }}$ determinations is $\sin ^{2} \theta_{\text {eff }}^{\text {lept }}=0.23153 \pm 0.00016$, with a $\chi^{2} /$ dof of $11.8 / 5$, corresponding to a probability of $3.7 \%$. The enlarged $\chi^{2} /$ dof is solely driven by the two most precise determinations of $\sin ^{2} \theta_{\text {eff }}^{\text {lept }}$, namely those derived from the measurements of $A_{\ell}$ by SLD, dominated by the left-right asymmetry result, and of $\mathrm{A}_{\mathrm{fb}}^{0, \mathrm{~b}}$ at LEP. These two measurements differ by 3.2 standard deviations. This is a consequence of the same effect as shown in Figure 1 (right).

\section{Global Standard Model Analysis}

Within the framework of the SM, each pseudo observable is calculated as a function of five main relevant parameters, which are the running electromagnetic and strong coupling constant evaluated at the $\mathrm{Z}$ pole, $\alpha_{e m}$ and $\alpha_{S}$, and the masses of $\mathrm{Z}$ boson, top quark and Higgs boson, $M_{\mathrm{Z}}$, $M_{\mathrm{t}}, M_{\mathrm{H}}$. Using the Fermi constant $G_{\mathrm{F}}$ allows to calculate the mass of the $\mathrm{W}$ boson. The running electromagnetic coupling is represented by the hadronic vacuum polarisation $\Delta \alpha_{\text {had }}^{(5)}$, as it is this contribution which has the largest uncertainty, $\Delta \alpha_{\text {had }}^{(5)}=0.02758 \pm 0.00035$ [7]. The precision of the Z-pole measurements requires matching precision of the theoretical calculations. The dependence on $M_{\mathrm{t}}$ and $M_{\mathrm{H}}$ enters through radiative corrections. The predictions are calculated with the computer programs [8] TOPAZ0 and ZFITTER, which incorporate state-of-the-art calculations.

Using the Z-pole measurements of SLD and LEP-I in order to evaluate electroweak radiative corrections, the masses of the two heavy particles, the top quark and the $\mathrm{W}$ boson, can be predicted. The resulting $68 \%$ C.L. contour curve in the $\left(M_{\mathrm{t}}, M_{\mathrm{W}}\right)$ plane is shown in Figure 2 (right). Also shown is the contour curve corresponding to the direct measurements of both quantities at the Tevatron and at LEP-II. The two contours overlap, successfully testing the SM at the level of electroweak radiative corrections. The diagonal band in Figure 2 (right) shows the constraint between the two masses within the SM, which depends on the mass of the Higgs boson, and to a small extent also on the hadronic vacuum polarisation (small arrow labeled $\Delta \alpha$ ). Both the direct and the indirect contour curves prefer a low value for the mass of the SM Higgs boson.

The best constraint on $M_{\mathrm{H}}$ is obtained by analysing all data. This global fit has a $\chi^{2}$ of 17.8 for 13 degrees of freedom, corresponding to a probability of $16.6 \%$. The pulls of the 18 measurements entering the fit are shown in Figure 3 (left). The single largest contribution to the $\chi^{2}$ arises from the $\mathrm{A}_{\mathrm{fb}}^{0, \mathrm{~b}}$ measurement discussed above, with a pull of 2.8. The fit yields $M_{\mathrm{H}}=91_{-32}^{+45} \mathrm{GeV}$, which corresponds to a one-sided 95\% C.L. upper limit on $M_{\mathrm{H}}$ of $186 \mathrm{GeV}$ including the theory uncertainty as shown in Figure 3 (left). The fitted $M_{\mathrm{H}}$ is strongly correlated with the fitted hadronic vacuum polarisation (correlation of -0.51$)$ and the fitted top-quark mass $(+0.52)$. The strong correlation with $M_{\mathrm{t}}$ implies a shift of $20 \%$ in $M_{\mathrm{H}}$ if the measured $M_{\mathrm{t}}$ changes by $3 \mathrm{GeV}$ (about one standard deviation). Thus a precise measurement of $M_{\mathrm{t}}$ is very important. Also shown are the $\chi^{2}$ curves obtained with the more precise but theory-driven evaluation of $\Delta \alpha_{\text {had }}^{(5)}$ [9], yielding a correlation of only -0.2 with $M_{\mathrm{H}}$, or including the results obtained in low- $Q^{2}$ interactions: atomic parity violation [10], Moller scattering [11], and NuTeV's measurement of deep-inelastic lepton-nucleon scattering [12]; with the two former measurements in agreement with the expectations but the latter differing by 3 standard deviations. Both analyses yield nearly the same upper limits on $M_{\mathrm{H}}$. 

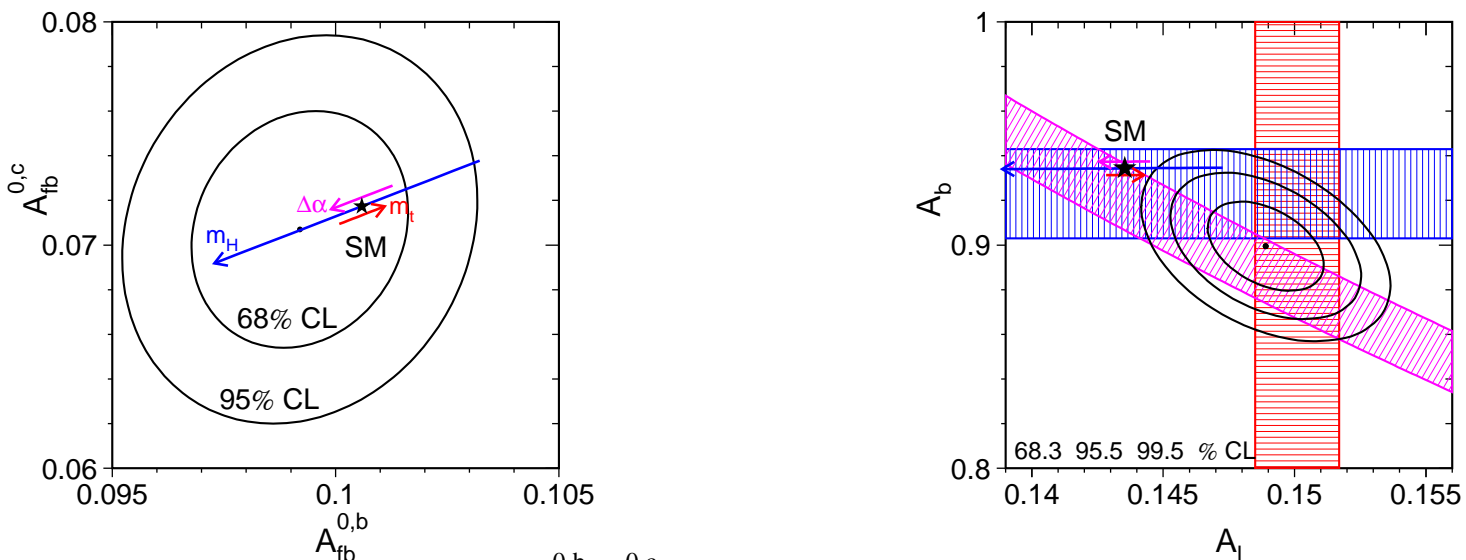

Figure 1: Left: Contour curves in the $\left(\mathrm{A}_{\mathrm{fb}}^{0, \mathrm{~b}}, \mathrm{~A}_{\mathrm{fb}}^{0, \mathrm{c}}\right)$ plane. Right: Bands of $\pm 1 \sigma$ showing the combined results of $\mathrm{A}_{\ell}, \mathrm{A}_{\mathrm{b}}$, and $\mathrm{A}_{\mathrm{fb}}^{0, \mathrm{~b}}=3 / 4 \mathrm{~A}_{\mathrm{e}} \mathrm{A}_{\mathrm{b}}$. The $\mathrm{SM}$ expectations are shown as the arrows for $M_{\mathrm{t}}=172.7 \pm 2.9 \mathrm{GeV}$ and $M_{\mathrm{H}}=300_{-186}^{+700} \mathrm{GeV}$ and $\Delta \alpha_{\text {had }}^{(5)}=0.02758 \pm 0.00035$.
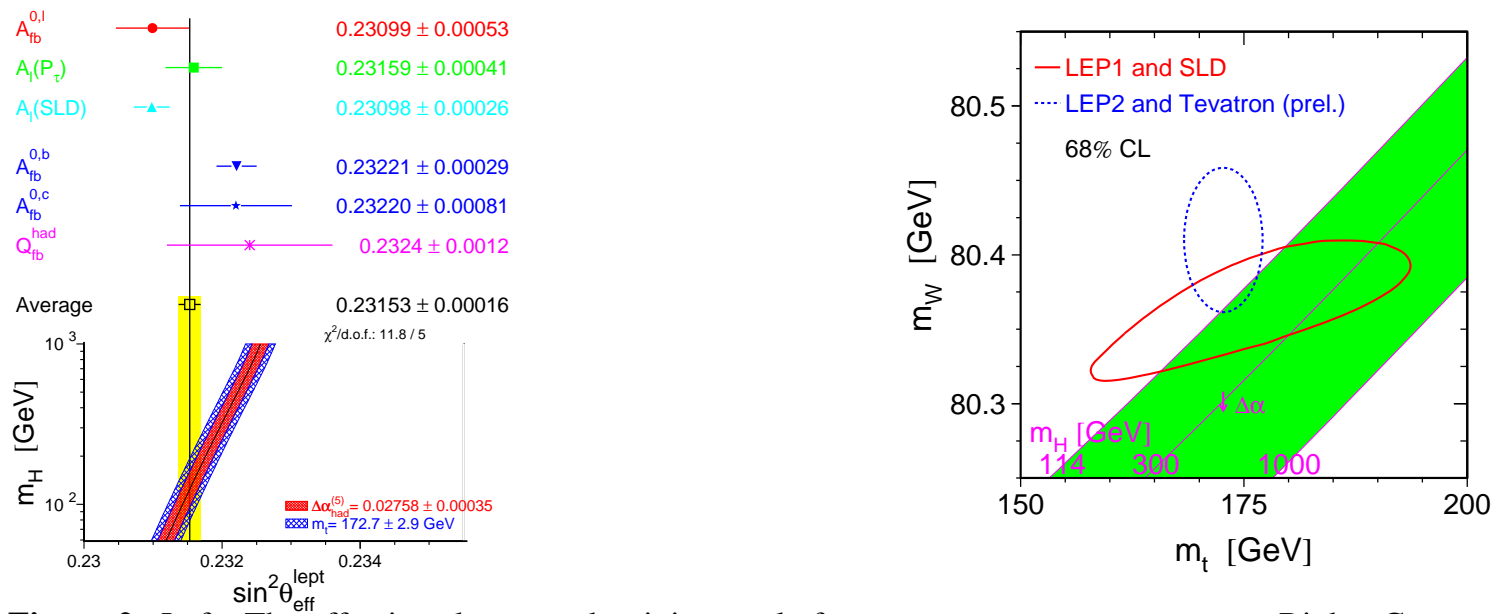

Figure 2: Left: The effective electroweak mixing angle from asymmetry measurements. Right: Contour curves of $68 \%$ C.L. in the $\left(M_{\mathrm{t}}, M_{\mathrm{W}}\right)$ plane for the direct measurements and the indirect determinations. The band shows the correlation between $M_{\mathrm{W}}$ and $M_{\mathrm{t}}$ expected in the SM.
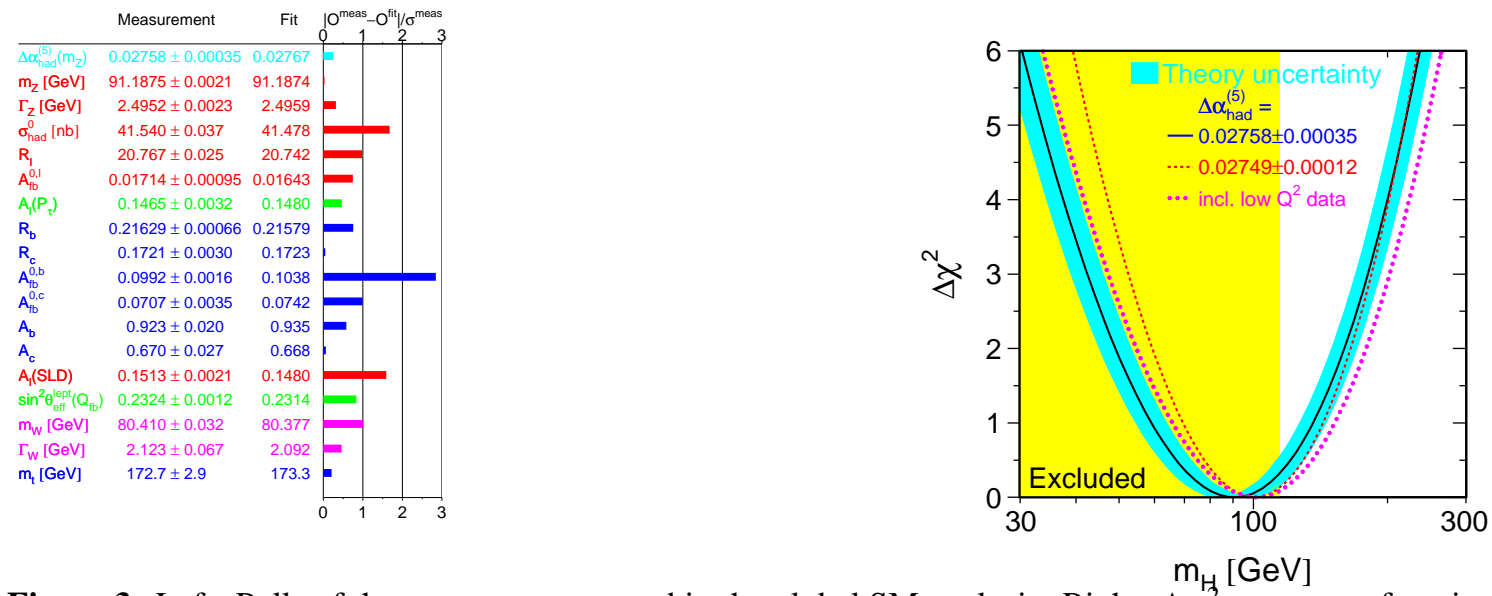

Figure 3: Left: Pulls of the measurements used in the global SM analysis. Right: $\Delta \chi^{2}$ curve as a function of $M_{\mathrm{H}}$. Also shown are the curves using a theory-driven evaluation of $\Delta \alpha_{\text {had }}^{(5)}$, or including the low- $Q^{2}$ measurements. 
The theoretical uncertainty on the SM calculations of the observables is visualised as the thickness of the blue band. It is dominated by the theoretical uncertainty in the calculation of the effective electroweak mixing angle, where a completed two-loop calculation is needed. The shaded part in Figure 3 (left) shows the $M_{\mathrm{H}}$ range up to $114.4 \mathrm{GeV}$ excluded by the direct search for the Higgs boson at $95 \%$ confidence level. Even though the minimum of the $\chi^{2}$ curve lies in the excluded region, the uncertainties on the Higgs mass value are such as that the results are well compatible.

\section{Conclusions}

During the last 15 years many experiments have performed a wealth of measurements with unprecedented precision in high-energy particle physics. These measurements test all aspects of the SM of particle physics, and many of them show large sensitivity to electroweak radiative corrections at loop level. Most measurements agree well with the expectations as calculated within the framework of the SM, successfully testing the SM at Born and at loop level. There are two "3 standard deviations effects", namely the spread in the various determinations of the effective electroweak mixing angle, within the SM analysis apparently disfavouring the measurement of $\mathrm{A}_{\mathrm{fb}}^{0, \mathrm{~b}}$, and NuTeV's result, most pronounced when interpreted in terms of the on-shell electroweak mixing angle. For the future, precise theoretical calculations including theoretical uncertainties are needed, in particular a completed two-loop calculation for the effective electroweak mixing angle. Experimentally, the next few years will bring improvements in the measurements of $\mathrm{W}$ and top masses, and the long-awaited discovery of the Higgs boson.

\section{Acknowledgements}

It is a pleasure to thank my colleagues of the Tevatron and LEP electroweak working groups, members of the E-158, NuTeV, SLD, ALEPH, DELPHI, L3, OPAL, CDF and DØ experiments, as well as T. Riemann and G. Weiglein for valuable discussions.

\section{References}

[1] LEP-EWWG, http://wWw. cern. ch/LEPEWWG.

[2] ALEPH, DELPHI, L3, OPAL, SLD, and the LEP-EWWG, hep-ex/0509008.

[3] Koji Sato, these proceedings.

[4] CDF, DØ and the TEV-EWWG, hep-ex/0507091.

[5] CDF, DØ and the TEV-EWWG, PRD 70 (2004) 092008.

[6] Ambreesh Gupta, these proceedings; Raimund Ströhmer, these proceedings.

[7] H. Burkhardt, B. Pietrzyk, PRD 72 (2005) 057501.

[8] G. Passarino et al., CPC 117 (1999) 278; D. Bardin et al., CPC 133 (2001) 229.

[9] J.F. de Troconiz, F.J. Yndurain, PRD 71 (2005) 073008

[10] J. Ginges, V. Flambaum, Phys. Rept. 397 (2004) 63.

[11] P. Anthony et al., E158 collaboration, PRL 95 (2005) 081601.

[12] G.P. Zeller et al., NuTeV collaboration, PRL 88 (2002) 091802, erratum 90 (2003) 239902. 